\title{
List of Cases
}

\section{International Courts}

Prosecutor $v$ Alex Tamba Brima, Bazzy Kamara and Santigie Borbor Kanu [2004] SCSL 16-T

Prosecutor $v$ Jean Paul Akayesu (Judgement) ICTY-94-4 of 2 September 1998

\section{Kenya}

C.K.W. v Attorney General \& another [2014] eKLR (Ochieng HC) Council of Imams and Preachers of Kenya, Malindi \& 4 others $v$ The Attorney General \& 5 others [2015] eKLR (Chitembwe HC)

Dennis Osoro Obiri v Republic [2014] eKLR (Kariuki, M'inoti, Mohammed CA)

David Mwangi Njoroge v Republic [2013] eKLR (G.W. NgenyeMacharia CA)

FIDA $v$ The Attorney General \& another [2018] eKLR (Mativo HC) Esther Nangwanaa Nandi v Jones Chewe Bobo [2006] eKLR (Rawal HC)

Esther Wanjiru Kiarie v Mary Wanjiru Githatu [2016] eKLR (Kimondo HC)

GMV v Bank of Africa Kenya Limited [2013] eKLR (Rika IC)

George Hezron Mwakio v Republic [2010] eKLR (Odero HC)

In the Matter of the Estate of the Late George Cheriro Chepkosiom (Deceased) [2017] eKLR (Ngugi HC)

Joan Bett v Republic [2017] eKLR (Mumbi Ngugi HC)

John Irungu v Republic [2016] eKLR (Asike-Makhandia, W. Ouko, K. M'inoti CA)

J.O.O. (also known as J.M.) v The Attorney General \& 6 others [2018] eKLR of 2014 (Ali-Aroni HC)

Joseph Lotoyo $v$ Republic [2011] eKLR

Katet Nchoe \& another v Republic [2011] eKLR (M.J. Anyara Emukule HC)

Pauline Robi Ngariba v Republic [2014] eKLR (D.S. Majanja HC) Peter Karumbi Keingati \& 4 others $v$ Ann Nyokabi Nguithi \& 6 others [2014] eKLR (Kimaru HC) 
Peter Mburu Echaria v Priscilla Njeri Echaria [2007] ${ }^{1}$ eKLR (Tunoi, O’Kubasu, Githinji, Waki, Deverell CA)

P K M v R P M [2017] eKLR (Okwengu, Musinga, GatembuKairu CA)

MBO v Republic [2010] eKLR (Omolo, Waki, Visram CA)

$M G N K v A M G$ [2016] eKLR (Karanja, Warsame, Azangalala CA)

$M A O$ \& another $v$ AG \& others [2015] eKLR (Ngugi HC)

Ngoka v Madzomba [2002] eKLR (Waki HC)

NML v Peter Petrausch [2015] eKLR (Rika IC)

$P N N v Z W N$ [2017] eKLR (Waki, Kiage, Azangalala CA)

P.O. $v$ Board of Trustees, AF \& 2 others [2014] eKLR (Rika IC)

P.O.O. (A Minor) v DPP \& another [2017] eKLR (Omondi HC)

Republic v Jackline Kwamboka Omboji [2018] eKLR (Okwany HC)

Robert Mutungi Muumbi v Republic [2015] eKLR

R P M v P K M [2015] eKLR (Kimaru HC)

Sheikh Ali Samoja v Republic [2016] eKLR (Odero HC)

W.J. and L.N. v Astarikoh \& 9 others [2011] eKLR (Ngugi HC)

C.K. (A Child) through Ripples International as her guardian

and next friend \& 11 others $v$ Commissioner of Police/Inspector

General of the National Police Service \& 3 others [2012] eKLR (Makau HC)

\section{Rwanda}

Mukamusoni Catherine v Mukagasana Domitilla [2013] RWSC 0087 of 2012 (Francine SC)

Mujawimana et al. v Bank of Kigali Ltd (BK) [2016] RWSC 0008 of 2014 (Kayitesi, Kayitesi, Nyirandabaruta SC)

Prosecution v Cyuma Miruho [0142/10/CS] RWSC (Rugege, Mukanyundo, Rugabirwa SC)

Prosecution v Fatirakumutima [2013] RWSC (Nyirinkwaya, Havugiyarmye, Mukamulisa SC)

Prosecution v Gatare [2014] RWSC 0317 (Immaculee, Fabien, Innocent SC)

Prosecution v Habimana [2010] RWSC 0321 [2016] (Mugenzi, Nyirinkwaya, Rugabirwa SC)

Prosecution v Maniragaba [2015] RWSC 0257 (Hatangimbabazi, Kalimunda, Munyangeri SC)

Prosecution v Mpitabakana [2014] RWSC 0129 (Nyirinkwaya, Havugiyaremye, Mukamulisa SC)

Prosecution v Naramabuye [2014] RWSC 0071 (Mutashya, Hitiyaremye, Kanyange SC) 
Prosecution v Ngurinzira [2015] RWSC (Mutashya, Munyangeri, Hitiyaremye SC)

Prosecution v Nkinamubanzi [2016] RWSC 0067 (Nyirinkwaya, Munyangeri, Hitiyaremye SC)

Prosecution v Nshimiyimana [2016] RWSC 00332 (Claire SC)

Prosecution $v$ Nshutirakiza [2015] RWSC 0047 of 2011 (Mutashya, Gakwaya, Hitiyaremye SC)

Prosecution v Uwiringiyimana [2017] RWSC 0067/13 (Mutashya, Rugabirwa, Kalimunda SC)

Prosecution v Uwizeye Eustache [2017] RWSC 0225 of 2013 (Mutashya, Rugabirwa, Karimunda SC)

RE $v$ N.J [2015] RWHC 0787 (Kaliwabo, Mukakalisa, Kabagambe $\mathrm{HC}$ )

Rutabayiru v Batamuliza [2016] RWSC 0013 (Mugenzi, Gakwaya, Munyangeri SC)

\section{Tanzania}

Agnes Doris Liundi v Republic [1980] TZCA 46 (Mustafa, Mwakasendo, Kisanga, CA)

Bashford v Tuli [1971] TZHC 76 (Hamyln HC)

Chalimba Chimbalemba v Republic [2011] Criminal Appeal No. 368 of 2008

Diha Matofali v Republic [2015] TZCA 245 (Kimaro, Mugasha, Mziray CA)

DPP v Jamal Waziri [2001] TZHC 324 (Munuo HC)

Edson Simon Mwombeki v Republic [2016] TZCA 94 (Rutakangwa, Massati, Mugasha CA)

Jenesia Philemon v Republic [2011] TZCA 179 of 2009 (Munuo, Nsekela, Mandia CA)

John Martin alias Marwa $v$ Republic [2017] Criminal Application No. 20 of 2014 (Mmilla and Mkuye CA)

Lawama Dedu v Republic [2016] Criminal Appeal No. 318 of 2015 (Mjasiri, Juma, Mugasha CA)

Leonard Jonathan $v$ Republic [2007] TZCA 225 (CA)

Mwita Masabo v Republic [2002] TZCA 56 (Lubuva, Mroso, Kaji CA)

Nyakanga v Mehego [1971] TZHC 270 (El Kindy HC) 
Rebeca Z. Gyumi v The Attorney General [2016] TZHC 5 (Munisi, Lila, Kihio HC)

Republic $v$ Modest \& another [1968] TZHC 26 (Seaton HC)

Thomas Adam v Republic [2012] TZCA 134 of 2010 (Kimaro, Mandia and Kaijage CA)

\section{Uganda}

Adoli Dickens v Uganda [2017] COA Criminal Appeal No. 41 of 2010 (Kakuru, Egonda Ntende, Obura CA)

Bizimana Jean Claude v Uganda [2014] COA Criminal Appeal No. 143 of 2010 (Kasule, Mwangusya, Egonda Ntende CA)

Bruno Kiwuwa v Ivan Serunkuma and Juliet Namazzi [2007] UGHC 52 of 2006 (Kasule HC)

Candia Akim v Uganda [2016] COA Criminal Appeal No. 181 of 2009 (Kasule, Obura, Byabakama-Mugenyi CA)

CEHURD \& 3 others $v$ The Attorney General [2011] UGCC 16 (Mpagi-Bahigeine, Byamugisha, Kavuma, Nshimye, Kasule CC) CEHURD \& 3 others $v$ The Attorney General [2013] UGSC 16 (Katureebe, Esther, Tumwesigye, Odoki, Tsekooko, Okello, Kitumba SC)

Diku Francisko v Uganda [2010] UGCA 304 (Kasule, Obura, Mugenyi CA)

Law and Advocacy for Women in Uganda $v$ The Attorney General [2010] UGCC 8 of 2007 (Mukasa-Kikonyogo, Mpagi-Bahigeine, Twinomujuni, Byamugisha, Kavuma CC)

Livingstone Ssewanyana v Uganda [2010] UGSC 19 of 2006 (Tsekooko, Katureebe, Kitumba, Tumwesigye, Kisaakye SC)

Juuko Musa v Uganda [2010] UGCA 180 (Kavuma, Buteera, Ntende CA)

Kaserebanyi James $v$ Uganda [2018] UGSC 10 of 2014 (Katureebe, Tumwesigye, Arach-Amoko, Mwangusya, Ekirikubinza SC)

Kooky Sharma and Anor v Uganda [2011] UGSC 44 (Odoki, Oder, Tsekooko, Karokora, Kanyeihamba SC)

Mifumi and Ors $v$ The Attorney General and Kenneth Kakuru [2014] UGSC 2 (B.M. Katureebe, Kisaakye, Tumwesigye, Odoki, Tsekooko, Okello, Kitumba SC)

Mureeba Janet \& 2 others $v$ Uganda [2002] UGSC 007 (Oder, Tsekooko, Karokora, Mulenga, Kanyeihamba SC) 
Muhwezi Alex and Hassan Bainomugisha v Uganda [2010] UGSC of 2005 (Tsekooko, Katureebe, Kitumba, Tumwesigye, Kisaakye, Ekirikubinza SC)

Nfutimukiza Isaya $v$ Uganda [2000] UGCA 41 of 1999 (Kato, Okello, Kitumba CA)

Ntambala Fred v Uganda [2018] UGSC 34 of 2015 (Tumwesigye, Mwangusya, Opio-Aweri, Mwondha, Ekirikubinza SC)

Otema David v Uganda [2015] UGCA 155 of 2008 (Mwangusya, Butera, Ntende CA)

Opira Mathew v Uganda [2000] UGCA 114 of 1999 (Kato, Okello, Engwau CA)

Kato Sula v Uganda [2000] UGCA 30 of 1999 (Kato, Okello, Kitumba CA)

Senyondo Wilson v Uganda [2003] UGCA 40 (MukasaKikonyogo, A. Twinomujuni, C.K. Byamugisha CA)

Ssendyose Joseph v Uganda [2010] UGSC 150 (Kasule, Aweri, Kakuru SC)

Uganda v Abiriga Michael alias Mayia \& another [2016] UGHC 0094 (Mubiru HC)

Uganda $v$ Akandinda Jackson [2014] UGHC 69 (Aweri-Opio HC)

Uganda $v$ Amoko [2014] UGHC 69 (Nahamya HC)

Uganda $v$ Anyolitho Denis [2016] UGHC 0182 (Mubiru HC)

Uganda v Balikamanya [2014] UGHC 25 of 2016 (Masalu HC)

Uganda v Dimba Pascal [2017] UGHC 89 of 2014 (Mubiru HC)

Uganda $v$ Eriya Paul [2009] UGHC 0010 (Nahamya HC)

Uganda $v$ Kakaire \& others [2015] UGHC (Nahamya HC)

Uganda v Kasujja Ivan [2014] UGHC 0004 (Wolayo HC)

Uganda v Kiryagana Emmanuel [2011] UGHC (Anglin HC)

Uganda $v$ Kusemererwa Julius [2015] UGHC 15 of 2014 (Batema HC)

Uganda v Muhwezi Lamuel [2012] UGHC 292 of 2010 (Kwesiga $\mathrm{HC}$ )

Uganda v Mukiibi Godfrey [2014] UGHC 0008 (Wolayo HC) Uganda v Mujuuzi Kalooli [2009] UGHC 116 (Nahamya HC)

Uganda v Muwanga Sepuya James [2016] UGHC 0108 (Mubiru HC) Uganda v Mwanje Aggrey [2015] UGHC 03 (Luswata HC) Uganda v Najja Sebango [2012] UGHC 65 (Nahamya HC) Uganda v Natukunda Faith [2012] UGHC 001 (Nahamya HC) Uganda v Nyanzi David [2016] UGHC 116 (Nahamya HC) Uganda v Okello Steven [2015] UGHC 093 (Kazibwe HC) Uganda v Okuni Dennis [2012] UGHC 0025 (Nahamya HC) 
Uganda v Okwera James [2016] UGHC 0004 (Nahamya C) Uganda v Olega Muhamad [2016] UGHC 33 (Mubiru HC)

Uganda $v$ Tumwesigye Zirabwa alias Sigwa [2013] UGHC 92 of 2011 (Namundi HC)

Uganda v Yiga Hamidu [2004] UGHC 5 of 2002 (Musoke HC)

\section{Note}

1 The wife, Priscilla Njeri Echaria, presented a complaint to the African Commission on Human and Peoples' Rights via Communication 375/09 (represented by Federation of Women Lawyers, Kenya and the International Centre for the Legal Protection of Human Rights). This was declared inadmissible for failure to comply with Article 56(6) of the ACHPR. 